\title{
BALANCES A QUARTZ RÉSONNANTS DE GRANDE SENSIBILITÉ FONCTIONNANT EN ULTRAVIDE ET A TRẼS BASSE TEMPÉRATURE : APPLICATION A L'ÉTUDE DES INTERACTIONS GAZ-SOLIDES
}

\author{
R. NUVOLONE et C. BOIZIAU
}

Service de Physique Atomique, Section d'Etudes des Interactions Gaz-Solides, Cen/Saclay BP no 2, 91-Gif-sur-Yvette (France)

(Reçu le 23 décembre 1971)

\begin{abstract}
Résumé. - Nous décrivons un dispositif expérimental destiné à l'étude des interactions entre les gaz et les solides à très basse température.

Le système de mesure proprement dit est constitué de six quartz résonnants. Cinq quartz très sensibles à la masse déposée sur leurs électrodes, permettent de mesurer la variation du nombre d'atomes condensés; le sixième est plus particulièrement sensible aux variations de température.

Les performances de l'appareil sont illustrées par l'étude de la sublimation du krypton solide.
\end{abstract}

\begin{abstract}
We describe an experimental apparatus used to study the interactions between gases and solids at very low temperatures.

The measuring system consists of six vibrating quartz crystals. Five of them very sensitive to the mass deposited on their electrodes, allow the variation of the number of condensed atoms to be measured ; the last one being particularly more sensitive to temperature variations.

The performance of the apparatus is illustrated with the study of solid krypton sublimation.
\end{abstract}

I. Introduction. - Les capteurs habituellement utilisés pour la mesure de concentration moléculaire fonctionnent en phase gazeuse (manomètres à ionisation-spectromètres de masse). Non seulement ils sont sensibles à tous les phénomènes qui peuvent se produire dans cette phase (rebondissement des atomes sur les parois - désorption des atomes ou molécules adsorbés sur les surfaces), mais ils risquent d'introduire des phénomènes parasites (bruit de fondpompage propre-effet thermique des filaments chauds).

De ce fait, leur emploi demeure délicat et il nous a semblé utile de développer une autre méthode de mesure. Notre choix s'est arrêté sur les balances à quartz résonnant, qui présentent l'avantage de donner des informations directes sur la surface où les phénomènes étudiés prennent naissance. De plus, nous montrerons plus loin que cette technique permet des mesures extrêmement fines de la variation de masse de la phase condensée $\left((\mathrm{d} m / \mathrm{d} t)_{\min }=10^{-12} \mathrm{~g} / \mathrm{s}\right)$.

Ces balances sont disposées comme le représente la figure 1 et rendent possibles, en particulier :

- la détermination de la tension de vapeur d'un cristal de gaz en présence de sa vapeur,

- l'étude de la distribution suivant trois directions de la sublimation des gaz solidifiés,

- l'étude de la réflexion suivant les trois mêmes directions d'un faisceau atomique ou moléculaire sur un cristal de gaz condensé.

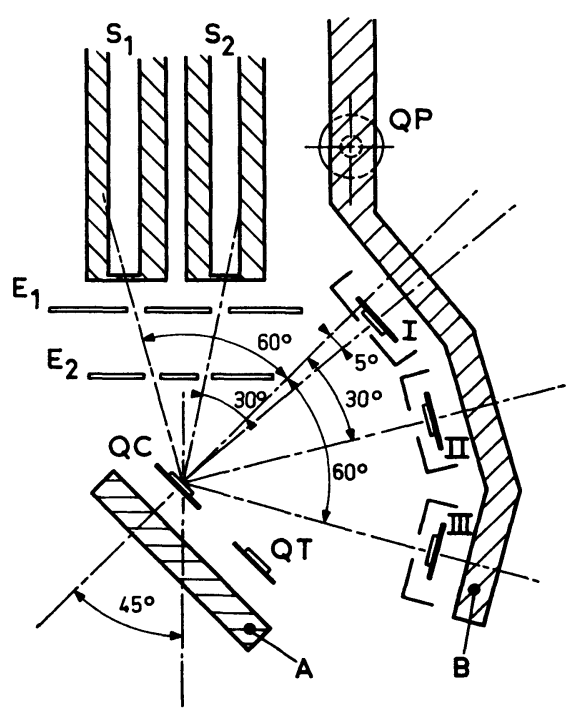

Fig. 1. - Schéma de la cellule expérimentale : $S_{1}, S_{2}$, sources du faisceau atomique ou moléculaire, dont les incidences $\left(60^{\circ}\right.$ et $30^{\circ}$ ) sont définies par les écrans $\mathrm{E}_{1}$ et $\mathrm{E}_{2} ; \mathrm{A}$, support de la balance cible $Q_{c}$ et du quartz température $Q_{T} ; B$, support des trois balances témoins, I, II, III et du quartz pilote $Q_{P}$.

II. Dispositif expérimental. - Il se compose de cinq parties principales (Fig. 2) :

- un système d'ultravide,

- un dispositif cryogénique, 


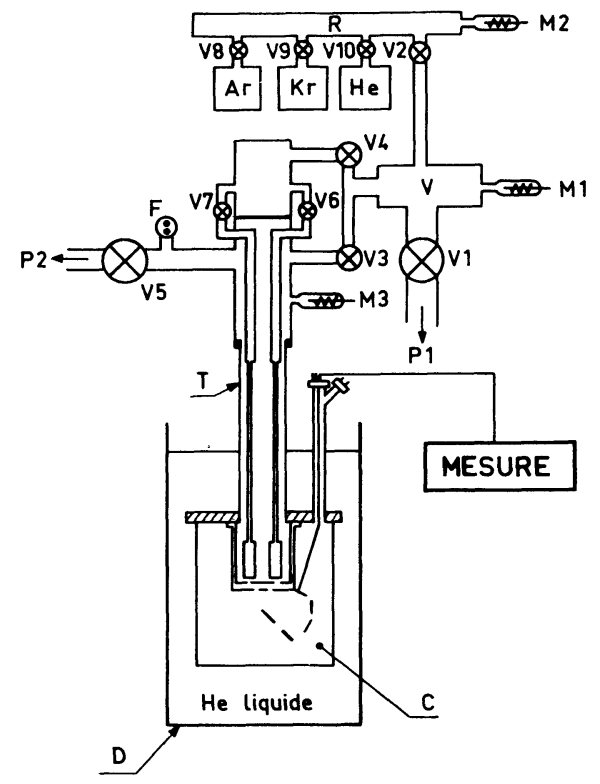

FIg. 2. - Schéma de principe du dispositif expérimental : C, cellule expérimentale (voir Fig. 1) ; D, dewar à Hélium liquide ; $F$, filtre de masse quadripolaire ; $M_{1}, M_{2}, M_{3}$, manomètres à ionisation de type Bayard-Alpert ; $\mathrm{P}_{1}$, colonne de pompage classique ; $P_{2}$, pompe ionique ; $R$, rampe de distribution des gaz purs; $T$, tube en paroi mince $V$, volume ; d'expansion du gaz étudié ; $\mathrm{V}_{1}$ à $\mathrm{V}_{10}$ : vannes étuvables.

- un circuit d'injection du gaz étudié, qui comporte deux sources de faisceau atomique et leurs organes de commande,

- la cellule de mesure,

- l'appareillage électronique.

2.1 SYStÈme D'UltRAVIDE. - Il est entièrement construit en acier inoxydable, l'étanchéité étant assurée par joints toriques en or. Cette technologie permet un étuvage destiné au dégazage de l'installation, ou après plusieurs utilisations successives, à l'élimination du gaz étudié adsorbé sur les parois.

L'étanchéité entre la cellule $\mathrm{C}$ et le tube $\mathrm{T}$ est obtenue par un joint en téflon de $0,2 \mathrm{~mm}$ d'épaisseur, serré par des vis en laiton, afin de bénéficier des effets de la dilatation thermique différentielle. Du fait de l'existence de ce joint nous limitons la température d'étuvage à 250 ou $300^{\circ} \mathrm{C}$; la durée de cette opération est portée à une semaine, afin que les gaz résiduels aient une pression négligeable devant celle du gaz injecté.

Le contrôle de ce vide résiduel est effectué à l'aide $\mathrm{du}$ spectromètre de masse quadripolaire $\mathrm{F}$, qui sert également à la mesure de la pureté du gaz injecté.

L'évacuation des gaz est assurée par deux groupes de pompage $\mathrm{P}_{1}$ et $\mathrm{P}_{2}$ dont les fonctions sont différenciées :

- $\mathrm{P}_{1}$, constitué d'une colonne classique (pompe primaire à palettes - pompe secondaire à diffusion d'huile silicone, dont les vapeurs sont condensées par un piège à effet Peltier puis par un piège à azote liquide) permet le démarrage de l'expérience, le main- tien d'une pression suffisamment basse durant l'étuvage, et l'évacuation du gaz étudié,

- $\mathrm{P}_{2}$ est une pompe ionique qui assure dans $\mathrm{C}$ une pression de base de l'ordre de $10^{-6} \mathrm{~N} . \mathrm{m}^{2}$.

Les pressions aux différents niveaux $M_{1}, M_{2}, M_{3}$ sont contrôlées par des manomètres à ionisation du type Bayard Alpert (courant électronique de $100 \mu \mathrm{A}$ ).

2.2 DisPoSITIF CRYOGÉNIQUE. - Sa partie principale est un Dewar cylindrique D superisolé en acier inoxydable.

Il comporte un anneau de garde rempli d'azote liquide, dont le niveau est maintenu constant à $10 \mathrm{~mm}$ près par une alimentation automatique. La cellule $\mathrm{C}$ étant placée au centre du Dewar $\mathrm{D}$, on ferme celui-ci par une plaque relativement étanche destinée à limiter la vaporisation de l'hélium liquide.

Le niveau d'hélium qui entoure la cellule $\mathrm{C}$ est maintenu constant à $\pm 5 \mathrm{~mm}$ près par une alimentation automatique commandée par sondes thermorésistantes.

En plus de sa fonction principale qui est d'amener les balances à une température telle que les gaz s'y condensent, ce dispositif cryogénique est aussi une cryopompe assez efficace pour maintenir dans $\mathrm{C}$ une pression très basse (inférieure à $10^{-8} \mathrm{~N} . \mathrm{m}^{-2}$ ). L'effet parasite de l'interaction du gaz environnant sur les surfaces étudiées se trouve donc réduit à un niveau négligeable.

Nous avons apporté une attention particulière au choix et à la disposition des différentes pièces qui constituent la cellule $\mathrm{C}$; ce qui a permis de limiter la consommation en hélium liquide à 1 litre toutes les $30 \mathrm{mn}$ d'exploitation.

2.3 Circuit D'INJECTION DES GAZ. - Il est constitué comme le montre la figure 2 :

- d'une rampe $\mathrm{R}$ sur laquelle sont connectées les bouteilles des divers gaz, par l'intermédiaire des vannes étuvables $V_{8}, V_{9}, V_{10}$,

- d'une vanne $V_{2}$ commandée par le manomètre $M_{2}$, qui permet de régler la pression du gaz à étudier,

- d'un volume $\mathrm{V}$ de 2 litres destiné à uniformiser la pression d'injection contrôlée par $\mathrm{M}_{1}$,

- de deux sources à effusion thermique $S_{1}$ et $S_{2}$ mises en service respectivement par $V_{6}$ et $V_{7}$.

Les sources $S_{1}$ et $S_{2}$ sont équipées sur leur pourtour de résistances chauffantes permettant à l'aide d'alimentations stabilisées de faire varier leur température de 50 à $300^{\circ} \mathrm{K}$, température contrôlée par des thermocouples en cuivre-constantan.

2.4 LA CELlULe DE MESURE. - La figure 1 en donne le schéma de principe :

Un tube central fixé à la bride du volume qui constitue la cellule de mesure maintient :

- d'une part les sources $S_{1}$ et $S_{2}$ et les écrans $E_{1}$ et $E_{2}$ qui définissent l'angle d'incidence du faisceau 
sur la cible $\mathrm{Q}_{\mathrm{C}}\left(60^{\circ}\right.$ pour $\mathrm{S}_{1}$ par rapport à la normale à $Q_{c}, 30^{\circ}$ pour $S_{2}$ ),

- d'autre part, les supports A et B sur lesquels seront fixées les balances à quartz résonnant.

Le support $\mathrm{A}$, incliné à $45^{\circ}$ par rapport à l'axe de symétrie de $S_{1}$ et $S_{2}$, porte le quartz $Q_{C}$, qui voit directement le faisceau atomique, ainsi qu'un quartz $Q_{T}$ qui, étant beaucoup plus sensible à la variation de température qu'à la variation de masse, mesure la température à la même distance du support que $Q_{C}$. Une résistance chauffante est brasée sur le pourtour de ce support ; un thermocouple en cuivre-constantan mesure les variations de température qu'elle provoque.

Le second support B, porte trois balances I, II, III qui mesurent dans le petit angle solide, qu'elles déterminent, le flux d'atomes réfléchis ou sublimés issus de $Q_{c}$.

Une balance à quartz pilote $Q_{p}$ est située sur le côté de $\mathrm{B}$, hors du trajet du faisceau et des gaz réfléchis (ou ré-émis). La constance de la fréquence de résonance de cette dernière donne la garantie de bonnes conditions expérimentales pour les paramètres pression-température.

Les axes de I, II, III font respectivement avec la normale à $\mathrm{Q}_{\mathrm{C}}$ un angle de $4^{\circ}, 30^{\circ}, 60^{\circ}$, tous trois étant situés dans le plan d'incidence du faisceau atomique. B est également équipé d'une résistance chauffante qui en permet le réchauffement et d'un thermocouple en cuivre-constantan.

Enfin, la cellule expérimentale est isolée de l'extérieur par une cloche en parois minces d'acier inoxydable.

III. Système électronique. - L'appareillage électronique attaché à l'ensemble expérimental peut être classé dans deux rubriques suivant ses fonctions :

- mesure des fréquences de résonance des balances à quartz,

- contrôle des températures.

3.1 MeSURE DES FRÉQUENCES. - Les balances à quartz résonnant ayant été décrites en détail par ailleurs [1], nous rappellerons simplement leurs caractéristiques dans le tableau I.

Chaque quartz est excité par un oscillateur, accordé pour la zone des températures de fonctionnement, sur la fréquence fondamentale de la balance (environ $5 \mathrm{MHz}$ ) et placé dans des conditions isothermes.

La mesure des fréquences s'effectue par l'intermédiaire d'un compteur digital $\mathrm{CD}$ connecté à un système de scrutation automatique dont le schéma de principe est représenté dans l'annexe I. Le signal recueilli par le système est soit envoyé sur une télétype $T$ qui restitue les grandeurs mesurées à la fois sous forme codée, sur bande pouvant être traitée par ordinateur (traitement en temps différé) et en clair sur imprimante, soit sur une chaîne d'acquisition et de traitement des données (traitement en temps réel). Le temps de comptage sur chaque quartz est ajustable de $10 \mathrm{~ms}$
TABleaU I

Caractéristiques générales

des balances à quartz réscinnonı

Cristal $\left\{\begin{array}{l}\text { coupe AT. } \varphi=40^{\circ} 10^{\prime}, \\ \text { lentille plan-convexe. Rayon de } \\ \text { courbure }: 300 \mathrm{~mm}, \\ \text { diamètre : } 15 \mathrm{~mm}, \\ \text { épaisseur : } 0,34 \mathrm{~mm} \text { au centre. }\end{array}\right.$

Electrodes $\left\{\begin{array}{l}\text { couches de chrome, argent, nickel, } \\ \text { forme circulaire, } \varnothing=6 \mathrm{~mm},\end{array}\right.$ épaisseur : $4000 \AA$.

ruban : bronze au Be ou acier inox,

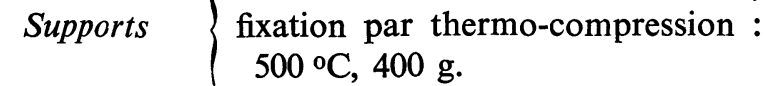

Caractéristiques
électriques $\left\{\begin{array}{l}f \simeq 5 \mathrm{MHz}, \\ \mathrm{R} \text { série }=2 \Omega, \\ \mathrm{L} \text { série }=81 \times 10^{-3} \mathrm{H}, \\ \mathrm{C} \text { série }=1,3 \times 10^{-14} \mathrm{~F}, \\ \mathrm{Q} \text { sous vide }=1,3 \times 10^{6}, \\ \mathrm{~N}=1670 \mathrm{kHz} / \mathrm{mm} .\end{array}\right.$

ANNEXE I

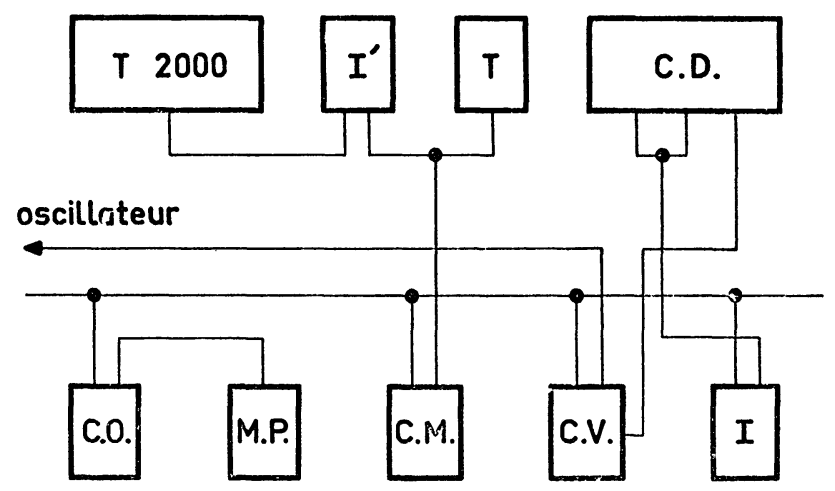

Schéma du système

de mesure automatique des fréquences

\section{ANALYSE D’UNE SÉQUENCE}

La matrice programme $M$. P. décode successivement les 256 instructions contenues dans le compteur ordinal $\mathrm{CO}$. - Le compteur ordinal $\mathrm{CO}$ demande à l'interface I :

1) une lecture du compteur $\mathrm{CD}$;

2) une conversion de la lecture.

- L'interface I envoie un signal de fin de lecture.

- Le compteur ordinal CO demande à la commande machine CM

- soit l'impression sur la télétype $\mathrm{T}$;

- soit la transmission vers l'ordinateur T 2000 , par l'interface $I^{\prime}$.

- Le compteur ordinal CO reçoit un signal de fin d' « impression ", revient au pas $\mathrm{O}$, demande la voie suivante au commutateur $\mathrm{CV}$.

- Le cycle recommence. 
à $90 \mathrm{~s}$, ce qui permet de mesurer des variations de $10^{-1} \mathrm{~Hz}$ à $10^{-3} \mathrm{~Hz}$ pour une fréquence voisine de $5 \mathrm{MHz}$.

Ainsi, compte tenu des caractéristiques des balances utilisées pour la période de comptage la plus longue $(90 \mathrm{~s})$, la plus petite variation de masse décelable est de $10^{-12} \mathrm{~g} . \mathrm{s}^{-1}$, ce qui correspond à une variation de l'ordre de $10^{-5}$ couche monoatomique de krypton par exemple.

Enfin, le glissement de fréquence mesurable est limité à $50 \mathrm{kHz}$ par la largeur de bande de l'oscillateur: la charge maximale des balances est donc d'environ de $10^{-3} \mathrm{~g}$.

3.2 CONTRôle DES TEMPÉRATURES. - Les températures des sources, des balances témoins et du support de la balance cible sont mesurées au moyen de thermocouples de cuivre-constantan. Chaque thermocouple a été étalonné aux basses températures. Afin de limiter les erreurs d'exploitation de la courbe d'étalonnage, nous avons déterminé son expression analytique jusqu'au second ordre. L'emploi d'un nanovoltmètre de grande sensibilité permet ainsi de mesurer des températures à $\pm 0,05^{\circ} \mathrm{K}$ entre $5^{\circ} \mathrm{K}$ et $125^{\circ} \mathrm{K}$.

La température au même niveau que le quartz cible est mesurée à l'aide d'un autre quartz résonnant. Ce cristal de type $\mathrm{AC}$ possède les mêmes caractéristiques que les balances à quartz résonnant; seul l'angle de coupe par rapport à l'axe optique est différent : $31^{\circ}$ au lieu de $40^{\circ} 10^{\prime}$.

Son coefficient de température $\alpha=(\Delta f / f) / \Delta T$ est environ égal à $2 \times 10^{-5}{ }^{\circ} \mathrm{C}^{-1}$, ce qui nous autorise à l'utiliser comme thermomètre. L'insertion de ce quartz dans le système de mesure des fréquences décrit plus haut permet après étalonnage, de mesurer la température dans la gamme $4^{\circ} \mathrm{K}$ à $273{ }^{\circ} \mathrm{K}$, à $\pm 0,05^{\circ} \mathrm{K}$.

IV. Evaluation du système expérimental. - 4.1. PRINCIPE DES MESURES. - La fréquence de résonance $f$ d'un quartz résonnant est inversement proportionnelle à la masse $m_{\mathrm{Q}}$ en vibration [1]

$$
\frac{\Delta f}{f}=-\frac{\Delta m}{m_{\mathrm{Q}}}
$$

soit

$$
\frac{\mathrm{d} m}{\mathrm{~d} t}=-\frac{m_{\mathrm{Q}}}{f} \frac{\mathrm{d} f}{\mathrm{~d} t} .
$$

L'éq. (1) donne la variation de masse sur le quartz dans l'unité de temps.

Lorsqu'un gaz de masse molaire $M$ se condense à la vitesse $\dot{n}_{\mathrm{c}}$ atomes. $\mathrm{cm}^{-2} \cdot \mathrm{s}^{-1}$, la variation de la masse par unité de temps est :

$$
\frac{\mathrm{d} m}{\mathrm{~d} t}=\dot{n}_{\mathrm{c}} \frac{M}{N_{\mathrm{a}}}
$$

avec $N_{\mathrm{a}}$ nombre d'Avogadro.
En combinant (1) et (2) on obtient :

$$
\dot{n}_{\mathrm{c}}=-\frac{N_{\mathrm{a}} m_{\mathrm{Q}}}{M f} \frac{\mathrm{d} f}{\mathrm{~d} t} .
$$

Dans notre cas : $f=5 \mathrm{MHz}, m_{\mathrm{Q}}=0,090 \mathrm{~g} \cdot \mathrm{cm}^{-2}$ d'où l'expression finale de $\dot{n}_{\mathrm{c}}$ :

$$
\dot{n}_{\mathrm{c}}=-\frac{1,08 \times 10^{16}}{M} \frac{\mathrm{d} f}{\mathrm{~d} t} \text { atomes. } \mathrm{cm}^{-2} \mathrm{~s}^{-1} .
$$

La mesure de la vitesse de condensation du gaz revient donc à déterminer la dérive de fréquence en fonction du temps (Fig. 3).

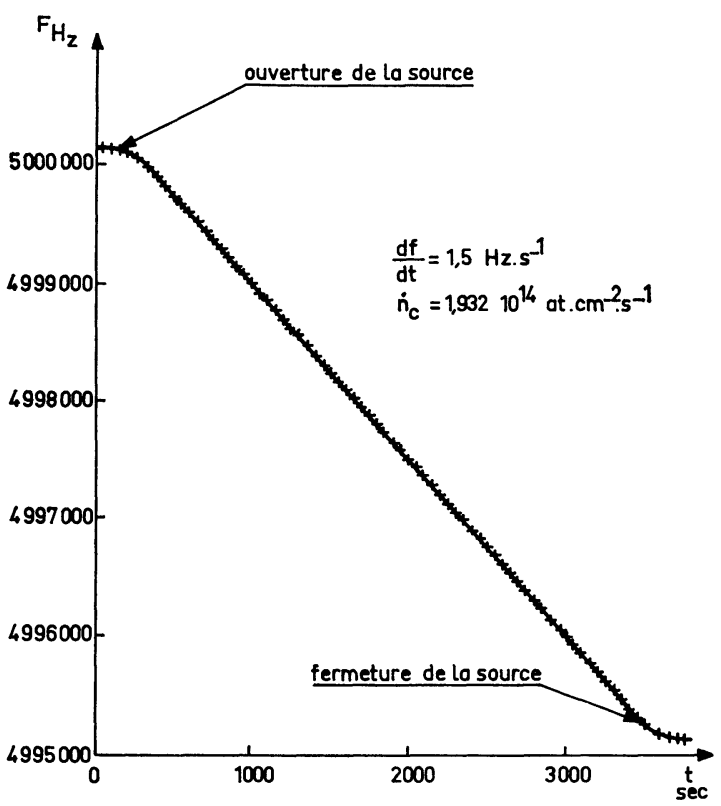

FIG. 3. - Construction d'un cristal de krypton.

Lorsque plusieurs centaines de couches de gaz ont été condensées sur $\mathrm{Q}_{\mathrm{C}}$, il est possible d'élever sa température jusqu'à une valeur que l'on maintient ensuite constante. La vitesse de sublimation $\mathrm{du}$ solide est obtenue de la même manière que la vitesse de condensation :

$$
\dot{n}_{\mathrm{s}}=-\frac{1,08 \times 10^{16}}{M} \frac{\mathrm{d} f}{\mathrm{~d} t} \text { atomes. } \mathrm{cm}^{-2} \mathrm{~s}^{-1} .
$$

A partir de (5) on calcule la tension de vapeur à la température de l'expérience en utilisant la formule classique :

$$
P=\dot{n}_{\mathrm{s}}(2 \pi m k T)^{1 / 2}
$$

où $m$ est la masse d'une molécule de gaz considéré.

Conjointement, les vitesses de condensation des atomes sur les balances I, II, III donnent le nombre de particules ré-émises dans ces directions.

Revenons à la figure 1 : lors d'une réflexion du faisceau sur $Q_{C}$, les vitesses de condensation sur $I$, II, III donnent cette fois le flux de gaz réfléchi suivant les directions déterminées par les axes des quartz. Il est également possible, en considérant la fraction 
condensée sur $\mathrm{Q}_{\mathrm{C}}$, de calculer le coefficient de capture du'gaz du faisceau incident à la température de celle-ci.

Dans ce dernier cas, il est nécessaire d'avoir au préalable étalonné les sources $S_{1}$ et $S_{2}$ en fonction du courant ionique délivré par le manomètre $M_{1}$ de la figure 1 , en maintenant $\mathrm{Q}_{\mathrm{C}}$ à une température assez basse pour que le coefficient de capture soit égal à 1 (Fig. 4).

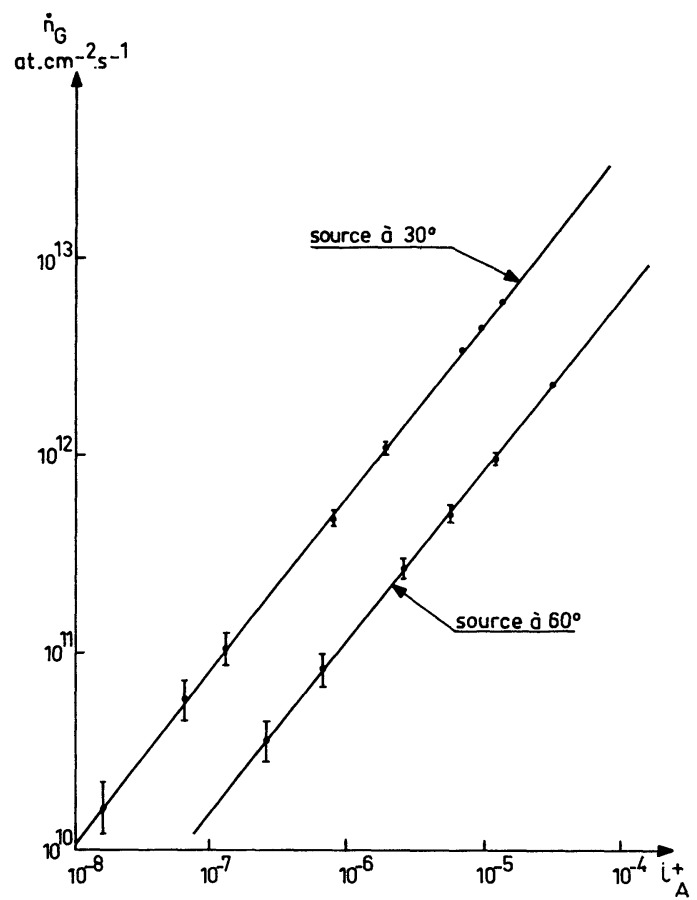

Fig. 4. - Etalonnage des sources pour le krypton, en fonction du courant ionique du manomètre $M_{1}$. Température du gaz dans la source : $125^{\circ} \mathrm{K}$.

4.2 MÉTHOde EXPÉRIMENTALE. - Afin d'illustrer les possibilités offertes par cette installation, nous avons choisi parmi les résultats déjà obtenus, de décrire les mesures effectuées sur la sublimation du krypton entre $30^{\circ} \mathrm{K}$ et $43^{\circ} \mathrm{K}$.

Le cristal de krypton a été obtenu par condensation sur l'électrode de $\mathrm{Q}_{\mathrm{C}}$ de 1500 monocouches de gaz fournies par la source $S_{2}$ (incidence de $30^{\circ}$ ) portée à $125^{\circ} \mathrm{K}$ (Fig. 3).

Le faisceau étant ensuite interrompu, nous observons l'évolution de la fréquence de résonance de $\mathrm{Q}_{\mathrm{C}}$ pour des paliers successifs de sa température.

Simultanément nous suivons l'évolution des fréquences des quartz I, II, III, afin d'obtenir trois points de la répartition spatiale des atomes sublimés.

4.3 RÉSULTATS EXPÉRIMENTAUX (SUBLIMATION DU KRYPTON. - a) Vitesse de sublimation du krypton. La vitesse de sublimation des atomes condensés sur $\mathrm{Q}_{\mathrm{C}}$ étant calculée à partir de la formule (1), la courbe expérimentale $\log _{10} n_{\mathrm{s}}=f\left(\frac{1}{T}\right)$ (Fig. 5) montre une partie rectiligne pour $T>35^{\circ} \mathrm{K}$, puis change de

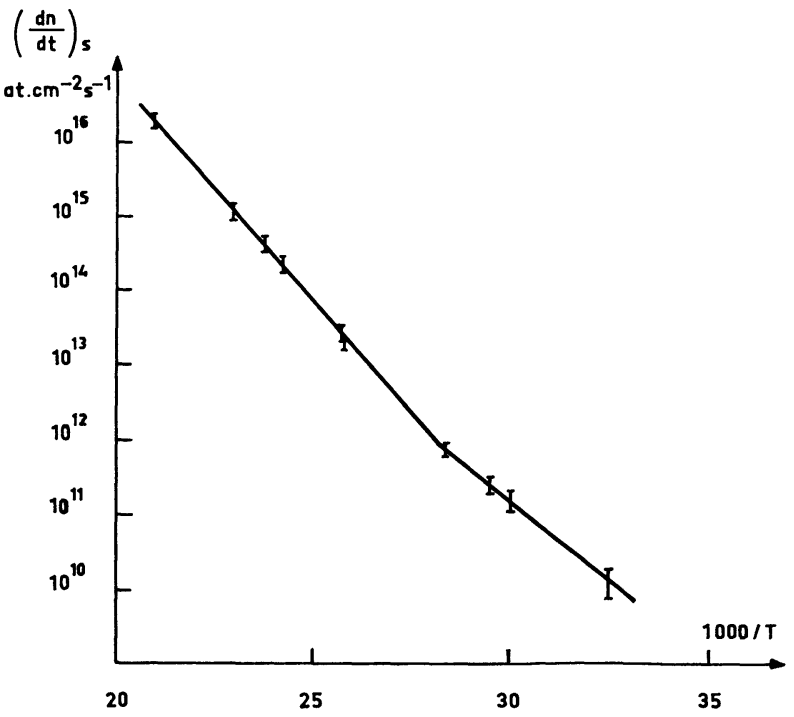

Fig. 5. - Vitesse de sublimation du krypton, en fonction de l'inverse de la température.

pente pour les températures plus basses. Considérant qu'au cours d'un processus de désorption $\dot{n}_{\mathrm{s}}$ est telle que $: \dot{n}_{\mathrm{s}}=\mathrm{d} m / \mathrm{d} t=A \mathrm{e}^{-E / k T}$ avec $E$ énergie d'activation de sublimation, $k$ constante de Boltzmann et $A$ une constante, la pente de la partie rectiligne donne l'énergie d'activation

$$
E=2410 \text { cal. mole }{ }^{-1} \pm 70 \text { cal. mole }{ }^{-1} .
$$

Cette valeur précise celle trouvée par L. L. Levenson sur un montage expérimental précédent [2].

b) Pression de vapeur saturante du krypton. A partir des valeurs expérimentales de $\dot{n}_{\mathrm{s}}$ et en utilisant la formule classique (6) nous avons calculé la pression de vapeur saturante du krypton dans la gamme $30^{\circ} \mathrm{K} \leqslant T \leqslant 43^{\circ} \mathrm{K}$. Les points expérimentaux, compte

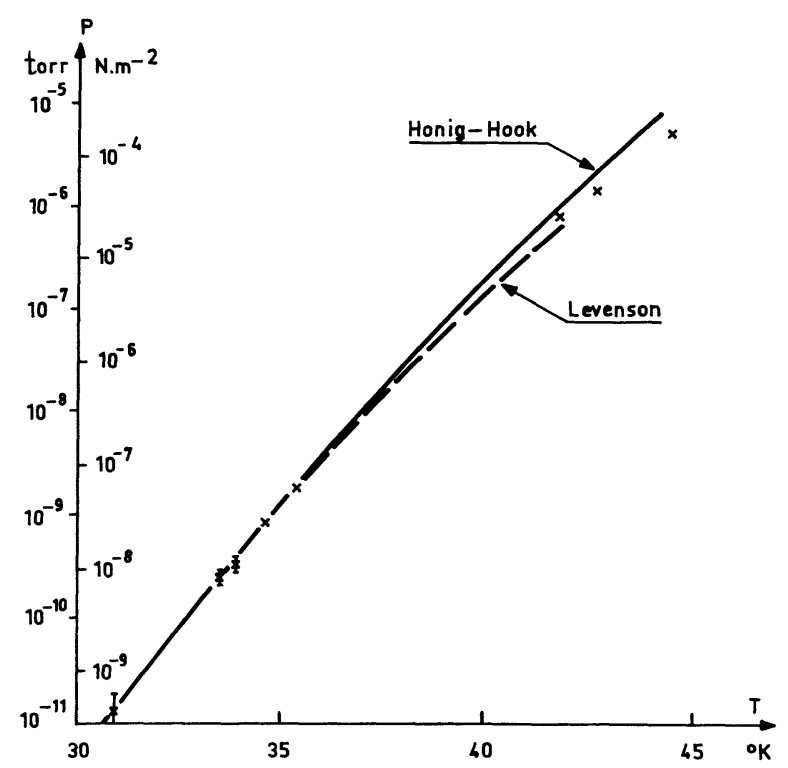

Fig. 6. - Tension de vapeur du krypton, en fonction de la température. 
tenu de la marge d'incertitude $\left( \pm 10^{-9} \mathrm{~N} . \mathrm{m}^{-2}\right)$ sont aussi en bon accord avec les résuitats de L. L. Levenson [2] et les extrapolations de Honig et Hook [3] (Fig. 6).

c) Répartition spatiale des atomes sublimés. La figure 7 donne pour chaque température explorée, la valeur de la dérive de fréquence en fonction de la direction d'observation.

Dans un but de comparaison, nous avons tracé

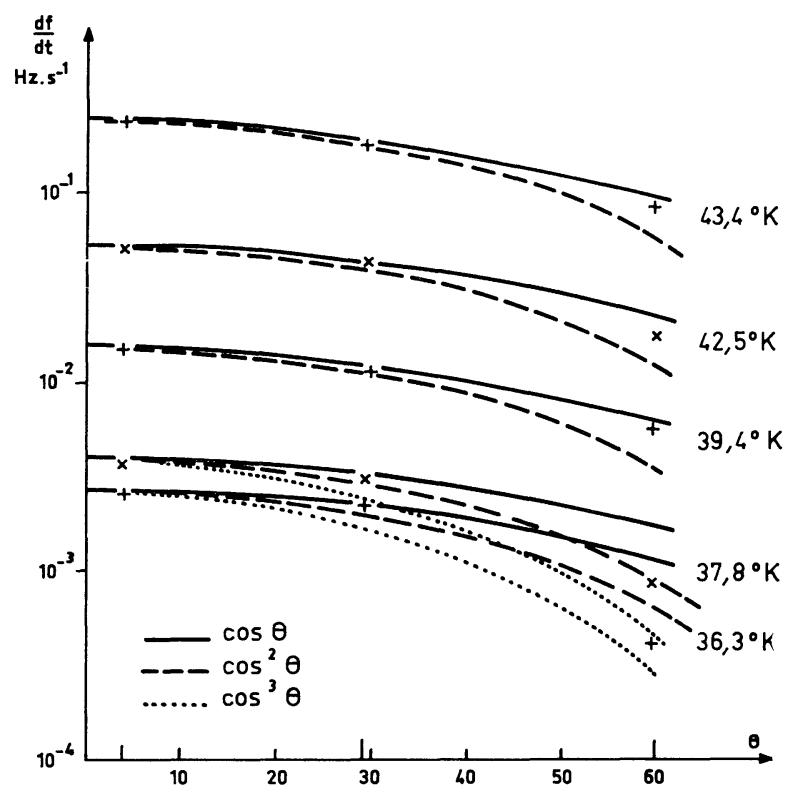

Fig. 7. - Répartition spatiale des atomes sublimés, comparée à des courbes en $\cos \theta, \cos ^{2} \theta, \cos ^{3} \theta$. des courbes représentant une distribution en cosinus (loi de Lambert), en cosinus carré et en cosinus cube. En première analyse, il semble que la ré-émission des atomes de krypton obéit à une loi située entre la loi de Lambert et une courbe en cosinus carré.

V. Conclusion. - Nous nous étions proposés de construire un dispositif expérimental utilisant à la fois les techniques des très basses pressions et des très basses températures, et destiné à l'étude de la sublimation d'un gaz condensé et de la diffusion d'un faisceau atomique ou moléculaire par une surface de gaz condensé.

Les balances à quartz résonnant nous ont permis d'utiliser une méthode d'approche, basée sur le relevé des informations sur la surface même où les phénomènes prennent naissance, en évitant de les perturber.

Les résultats expérimentaux obtenus justifient pleinement a posteriori cette méthode de mesure, compte tenu de la grande sensibilité dynamique des quartz $\left(10^{-12}\right.$ g. $\left.\mathrm{s}^{-1}\right)$, et de la souplesse d'emploi du système automatique d'acquisition des données.

VI. Remerciements. - Les auteurs tiennent à remercier tout particulièrement MM. Manus, Degras, Armand et Schram pour l'intérêt qu'ils ont porté à la réalisation de cet ensemble expérimental, M. Schmouchkovitch pour la mise au point des oscillateurs, M. Le Grand pour le développement des programmes de traitement automatique des résultats et Mme Lauret pour la dactylographie du manuscrit

\section{Bibliographie}

[1] Nuvolone (R.), Boiziau (C.), Réalisation d'une balance à quartz de grande sensibilité (séminaire sur le contrôle continu des dépositions sous vide, Genève, 21-22 juin 1971). A paraître dans «Le Vide ".

[2] Levenson (L. L.), Contribution à l'étude thermody- namique et cinétique de l'Argon, du Krypton et du Xénon à très basse température (Thèse $n^{0} 38$, Faculté des Sciences d'Orsay, juin 1968).

[3] Honig (R. E.), Hook (H. O.), Vapour pressure data for some common gases (RCA Review, 1960, 21, 360 . 